\title{
Editorial
}

\section{Por una política educativa más allá de la colonialidad del Ser, del Saber y del Poder \\ https://doi.org/10.19053/01227238.9409}

La Revista Historia de la Educación Latinoamericana ${ }^{1}$, en este número aborda el tema de las políticas para la Educación Superior en América Latina, reflexionando desde una perspectiva de la Historia de las Universidades en América y Europa, o más específicamente, en el espacio iberoamericano y se inicia con un artículo de España, en el que su autor aboga por una "nueva episteme" que venga a ventilar la institución Universitaria. Podríamos decir, que él reivindica una (re) invención de la Universidad en Iberoamérica, bajo nuevas perspectivas políticas.

El Profesor Justo Cuño y yo veníamos desde hace tiempo pensando plantear un número especial de la Revista de Historia de la Educación Latinoamericana (RHELA) de la Sociedad de Historia de la Educación Latinoamericana sobre políticas de educación superior en América Latina e Iberoamérica, en un momento en que dichas políticas han pasado por férreos ajustes y sumisión con respecto al capital financiero internacional. Efectivamente, desde finales del siglo veinte irrumpieron una serie de reformas que se consolidaron al inicio de este nuevo siglo a través de un modelo neoliberal impuesto y desarrollado dentro de la lógica del capital. Traemos para el debate varios ensayos e investigaciones sobre el tema, pues considerábamos que el momento es oportuno para hacer un balance de las políticas de la Educación Superior hasta el tiempo presente.

Respaldados por las reflexiones provocadas por los coloquios iberoamericanos sobre la historia de la universidad y los Congresos Internacionales sobre la Historia de las Universidades en América y Europa, realizados en el espacio iberoamericano, (con sus últimas ediciones en Carmona, Andalucía, y Temuco, Chile), estos foros se convirtieron en cuna de la discusión político-social sobre la universidad, tanto en el Grupo de Investigación HISULA (Historia a la Universidad Latinoamericana) como en SHELA (Sociedad de Historia de la Educación Latinoamericana). Esta agenda provocó en nosotros y en el grupo ${ }^{2}$ la necesidad de reflexionar de manera más orgánica la temática sobre las políticas universitarias en el espacio en que actuamos.

Por lo tanto, es en el marco de esta breve síntesis histórica que hemos sido invitados a organizar ese número sobre Política de Educación Superior en Ibe-

$1 \quad$ Ver artículos relacionados: Diana Soto Arango; Aracely Forero Romero. La Universidad Latinoamericana y del Caribe en los Desafíos del Siglo XXI. Revista Historia de la Educación Latinoamericana, vol. 18 no. 26 (2016): 279-309. https://doi. org/10.19053/01227238.4375; Justo Cuño Bonito. Reforma Y Contrarreforma de la Enseñanza Primaria Durante La II República Española y el Ascenso del Fascismo (1932-1943). Revista Historia de la Educación Latinoamericana vol. 15 no. 21 (2014): 89106. https://doi.org/10.19053/01227238.2468; Alejandra Contreras Gutiérrez. La enseñanza jesuita en Chile colonial: sus colegios, universidades y una aproximación a sus métodos y contenidos. Revista Historia de la Educación Latinoamericana, vol. 16 no. 22 (2014): 35-50. https://doi.org/10.19053/01227238.2687.

2 José Rubens Lima-Jardilino; Diana Soto Arango. (Org). Políticas Universitarias en Latinoamérica: Historia y Perspectiva. 1. ed. (São Paulo: Emblema 2006). 
roamérica. En él presentamos un dossier compuesto de siete (7) artículos sobre la temática y otras tres reflexiones que debaten las políticas en otros niveles de la Educación. Hemos reunido aquí textos de diferentes eventos promovidos por esas instituciones, los cuales fueron evaluados por pares y componen este compendio sobre políticas universitarias en Latinoamérica que tenemos el placer de presentar al lector.

El primer texto, al que ya se hizo mención anteriormente, se refiere al análisis macro del ataque de las políticas neoliberales a la universidad que vivimos hoy. El análisis del texto se orienta sobre las causas del fenómeno de globalización y la sujeción de los estados-nación a los dictámenes del capital. El autor nos propone una educación más allá del capital ${ }^{3}$, pero indica, como desafío, la posibilidad de construcción de un espacio iberoamericano del conocimiento como herramienta de construcción de una alternativa para pensar una "otra episteme", en los moldes de una epistemología del sur (considerando que sur no significa aquí solamente el sur geográfico, sino el geopolítico $\left.{ }^{4}\right)$. El artículo es una buena dosis de aji (picante) en nuestro cerebro para incomodarnos a un proceso reflexivo profundo sobre esa historia del Tiempo Presente ${ }^{5}$.

Los seis artículos siguientes, son estudios y reflexiones sobre políticas de la Educación Superior y universitarias en las diversas experiencias de Latinoamérica, a saber: Argentina, Colombia, Brasil, Venezuela, México.

La Dra. Cristina Vera Flachs hace un recorrido por las políticas educativas en Argentina, tomando como caso la Universidad de Córdoba en la construcción del marco legal para las políticas del siglo XX. Le siguen los trabajos de Sara Guerrero y Diana Soto Arango que hacen una reflexión desde Colombia tomando en cuenta la masificación de la educación superior y el abandono del Estado respecto a las Universidades públicas. Después tenemos el artículo de Celia Haas que nos impone una reflexión urgente y bastante compleja. Se trata del tema de la masificación de la educación superior en Brasil por medio de la llamada Educación a Distancia (EaD). Fenómeno este regulado, asumido e incentivado legalmente por el Estado brasileño y en los demás países del espacio latinoamericano, como herramienta para suplir el desequilibrio en la oferta de educación superior disponible para los jóvenes brasileños. La profesora Haas

3 István Mészarós. Más allá del capital. Hacia una teoría de la transición. Asamblea Legislativa Plurinacional de Bolivia, (La Paz, 2010).

4 Sobre el término SUR, estoy aquí apoyándome en la concepción utilizada por Sousa-Santos y utilizándolo metafóricamente como "un campo de desafíos epistémicos que busca reparar los daños e impactos históricamente causados por el capitalismo y su relación colonial con el mundo. Esta concepción de Sur presupone en parte sí al Sur geográfico, el conjunto de países y regiones del mundo sometidos al colonialismo (...). Pero, por otra parte también se refiere a un 'Sur' en el interior del Norte geográfico que se componen de clases y vastos grupos (trabajadores, mujeres, indígenas, afrodescendientes) que fueron sujetos a dominación capitalista y colonial. In. Sousa-Santos, Boaventura; Meneses, María Paula (2009) [org]. Epistemologias do Sul. Coimbra: Edições Almedina, 12 - 13.

5 En lo que pese a las resistencias del campo científico de la Historia y las recomendaciones de que el historiador debe distanciarse de los tiempos del acontecimiento de los hechos, para analizarlo con cierta exención, incluso después de la Escuela de los Anales, no se puede dudar de que hay hoy día una historiografía del tiempo presente en curso y cada vez más cerca de nosotros. Los historiadores han llamado la atención sobre la manera como las sociedades contemporánea están manejando el tiempo presente. Si a lo largo del siglo XIX los historiadores creían que las sociedades caminaban hacia el progreso y que el futuro se avecinaba como algo prometedor, en el contexto actual la creencia en el futuro se vuelve cada vez más incierta, transformando ese futuro en presente, un cierto 'presentismo'. Lo que se tiene de eso es un cuadro de una supervaloración de la memoria y de temas como identidades y conmemoraciones. Los testimonios ganan nuevas dimensiones. Una historia del tiempo presente que ahora no se encuentra solamente en los archivos, pero también en los testigos de ese tiempo. 
aborda el fenómeno y apunta críticamente sobre los males del modelo en lo que se refiere a la cobertura, evasión y poca calidad. El siguiente artículo, de las manos de dos schoolar bolivarianos, los profesores doctores Reinaldo Hojas y José Pascual Mora-García, traen para el lector un análisis del proyecto bolivariano de educación superior y los impactos de esta política en la Universidad y en la Investigación venezolana. Aunque abordan una reflexión de larga duración, el recorte en el que los autores se centran, está referido a la "Era Chaves". El siguiente artículo sobre la Educación y la Universidad en Colombia, escrito por Luis Alberto Malagón, Luz Helena Rodríguez y Diego Machado trae la reflexión sobre un tema bastante discutido en la actualidad tras el proceso de expansión de la universidad en América Latina, a saber: la cuestión de la calidad de la educación superior. Los autores toman como estudio de caso a Colombia. Coronando ese esfuerzo analítico del presente dossier, finaliza el bloque con el texto del profesor Armando Martínez Moya, profesor de la Universidad de Guadalajara y actual presidente de SHELA. El profesor Martínez dirige su reflexión hacia las políticas educativas que poco a poco provocaron el abandono y completo olvido de las humanidades en los estudios universitarios y las políticas públicas y toma como caso la Universidad de Guadalajara.

El segundo bloque está compuesto por artículos que también aportan reflexiones sobre las políticas educativas, pero dirigidas a otros niveles y modalidades de la educación. El primer texto nos provoca con un tema muy cercano a la Universidad latinoamericana en lo que se refiere a su decolonialidad del saber y del poder ${ }^{6}$ : discute el significado de la educación Aimara en Perú y las políticas que inciden en la lucha por una educación más intercultural. Aunque trata de la educación común a todos los ciudadanos Aimaras, se centra en la lucha continua por la implantación de una universidad intercultural (Aimara) y en la lucha por el acenso a la educación superior para los pueblos originarios. En este bloque contamos, además, con la reflexión del profesor Ilich Silva sobre las políticas educativas en Chile. Él elabora su análisis crítico a partir de los conceptos de regulación y desregulación del mercado en el proceso educativo en Chile y su repercusión para la formación docente. Otro estudio importante es el de Gustavo Correa y Juan David Parra sobre las políticas educativas y el sistema de educación básica en el Caribe, a través de una perspectiva historiográfica.

El artículo que abre el presente número de RHELA procede de Paraguay, lo que es una grata novedad para nuestro grupo HISULA, pues siempre habíamos reclamado una reflexión desde ese importante país sudamericano. Anahí Soto y David Velázquez abordan la investigación de la educación y su desarrollo en el siglo XX en Paraguay, a partir de la importante trayectoria del intelectual y educador paraguayo Ramón Indalecio Cardozo, pionero de la escuela activa en ese país y constructor de una reforma de la educación paraguaya que transformó sus más importantes paradigmas a principios del siglo XX.

6 Aníbal Quijano. Colonialidade do poder, Eurocentrismo e América Latina. En. A colonialidade do saber: eurocentrismo e ciências sociais. Perspectivas latino-americanas. (Buenos Aires: CLACSO, Consejo Latinoamericano de Ciencias Sociales. 2011) o http:// bibliotecavirtual.clacso.org.ar/clacso/sur-sur/20100624103322/12_Quijano.pdf 
Pensamos que ese número aportará una visión renovada en la cuestión de las políticas educativas en América Latina y el Caribe. Sin perder de vista, por supuesto, que necesitamos pensar en políticas educativas que colaboren para la superación del paradigma de la colonialidad y nos impulse hacia una decolonización del Ser, del Poder y del Saber, estas investigaciones nos ayudarán a pensar en una nueva episteme desde el sur, con vistas a salir de la subalternidad y alcanzar otro protagonismo, con otra educación, con otro saber, con un objetivo claro: construir una nueva epistemología desde el sur.

\section{Buena lectura}

Justo Cuño Bonito

Universidad Pablo de Olvide (España)

Grupo de investigación ILAC - UPTC

José Rubens Lima Jardilino

Universidade Federal de Ouro Preto (Brasil)

Grupo de investigación HISULA - UPTC

https://doi.org/10.19053/01227238.9409

\section{REFERENCIAS}

Contreras Gutiérrez, Alejandra. La enseñanza jesuita en Chile colonial: sus colegios, universidades y una aproximación a sus métodos y contenidos. Revista Historia de la Educación Latinoamericana, vol.16 no. 22 (2014): 35-50. https://doi.org/10.19053/01227238.2687.

Cuño Bonito, Justo. Reforma y Contrarreforma de la Enseñanza Primaria durante la II República Española y el Ascenso del Fascismo (1932-1943). Revista Historia de la Educación Latinoamericana vol. 15 no. 21 (2014): 89-106. https://doi.org/10.19053/01227238.2468

Lima-Jardilino, José Rubens; Soto Arango, Diana A., (Org). Políticas Universitárias en Latinoamérica: Historia y Perspectiva. 1. ed. (São Paulo: Emblema 2006).

Quijano, Aníbal. Colonialidade do poder, Eurocentrismo e América Latina. En. A colonialidade do saber: eurocentrismo e ciências sociais. Perspectivas latino-americanas. Buenos Aires: CLACSO, Consejo Latinoamericano de Ciencias Sociales. 2011.

Mészarós, István. Más allá del capital. Hacia una teoría de la transición. Asamblea Legislativa Plurinacional de Bolivia, La Paz. 2010.

Soto Arango, Diana; Forero Romero, Aracely. La Universidad Latinoamericana y del Caribe en los Desafíos del Siglo XXI. Revista Historia de la Educación Latinoamericana, vol. 18 no. 26 (2016): 279-309. https://doi.org/10.19053/01227238.4375 